The radical character of the acenes: A density matrix renormalization group study

Johannes Hachmann, Jonathan J. Dorando, Michael Avilés, and Garnet Kin-Lic Chan

Citation: J. Chem. Phys. 127, 134309 (2007); doi: 10.1063/1.2768362

View online: http://dx.doi.org/10.1063/1.2768362

View Table of Contents: http://aip.scitation.org/toc/jcp/127/13

Published by the American Institute of Physics 


\title{
The radical character of the acenes: A density matrix renormalization group study
}

\author{
Johannes Hachmann, Jonathan J. Dorando, Michael Avilés, and Garnet Kin-Lic Chan \\ Department of Chemistry and Chemical Biology, Cornell University, Ithaca, \\ New York 14853-1301, USA
}

(Received 21 May 2007; accepted 10 July 2007; published online 4 October 2007)

\begin{abstract}
We present a detailed investigation of the acene series using high-level wave function theory. Our $a b$ initio density matrix renormalization group algorithm has enabled us to carry out complete active space calculations on the acenes from napthalene to dodecacene correlating the full $\pi$-valence space. While we find that the ground state is a singlet for all chain lengths, examination of several measures of radical character, including the natural orbitals, effective number of unpaired electrons, and various correlation functions, suggests that the longer acene ground states are polyradical in nature. (C) 2007 American Institute of Physics. [DOI: 10.1063/1.2768362]
\end{abstract}

\section{INTRODUCTION}

The acenes are the series of ladderlike compounds consisting of linearly fused benzene rings (Fig. 1) ${ }^{1,2}$ Due to their technological potential ${ }^{3-5}$ and their intrinsic value as models for more complex conjugated molecules, they have been the subject of many theoretical and experimental investigations. ${ }^{6-14}$ In a number of recent studies, it has been proposed that longer acenes may possess an unusual electronic ground state that is not the simple closed-shell singlet suggested by molecular orbital arguments. Based on extrapolating the experimental singlet-triplet gap of the acenes up to pentacene, Angliker et al. ${ }^{7}$ predicted that the ground state of higher acenes from nonacene upwards would be a triplet. Density functional calculations by Houk et al. ${ }^{11}$ also predicted a singlet-triplet cross over. However, Bendikov et $a l^{12}$ noted that the restricted singlet density functional ground state would become unstable to an open-shell singlet, or singlet diradical, configuration for acenes longer than hexacene. The open-shell singlet-triplet gap for the longest acene studied (decacene) was estimated as ranging from $1.5[\mathrm{BLYP} / 6-31 \mathrm{G}(d)]$ to $5.7[\mathrm{~B} 3 \mathrm{LYP} / 6-31 \mathrm{G}(d)] \mathrm{kcal} / \mathrm{mol}$.

Despite these intriguing findings, the density functional results leave many interesting questions unanswered. For example, how diradicaloid are the acenes really compared to conventional diradical systems? As we go to longer acenes, might we expect to find triradical and even higher polyradical ground states? And if so, how do we understand the electronic structure and bonding in these states? Such questions, which probe the essential many-electron character of di- (and indeed poly) radicalism, are not easily answered through density functional theory based on a single Kohn-Sham determinant.

For this reason we have decided to explore the nature of the acene ground state using high-level wave-function-based electronic structure theory. The many-electron correlations in radical wave functions tell us about the coupled simultaneous motions of the electrons. Conceptually, singlet states with unpaired electrons require multiconfigurational wave functions $^{15-23}$ as used, for example, in the complete active space (CAS) family of methods. ${ }^{24}$ In the acenes, the ideal choice of active space would be the complete $\pi$-valence space, i.e., the set of all conjugated $p_{z}$ orbitals. However, the exponential cost of traditional CAS methods as a function of the number of correlated orbitals and electrons renders calculations with the complete $\pi$-valence space impossible for acenes much longer than napthalene, which already has ten conjugated orbitals and electrons. Consequently, earlier CAS calculations could only use an incomplete $\pi$-valence space. ${ }^{12,25}$

The density matrix renormalization group (DMRG) algorithm ${ }^{26-28}$ provides a way to overcome the traditional exponential complexity of CAS methods in long molecules such as the acenes. ${ }^{29,30}$ We have recently developed a local $a b$ initio DMRG method that computes an essentially exact CAS wave function with an effort that scales only quadratically with the length of the molecule. ${ }^{31}$ Consequently, we can now extend the range of traditional CAS calculations in long molecules from about 10 orbitals to 100 active orbitals or more. In the current work we apply our ab initio DMRG algorithm to the acene series from napthalene (2-acene) to dodecacene (12-acene), in all cases correlating exactly the complete $\pi$-valence space. First, we revisit the question of the relative stabilities of the singlet and triplet states. Then, using our correlated wave functions, we embark on a detailed study of the radical nature of these systems. We find intriguingly that the higher acenes are not only diradicals but possess increasing polyradical character. By explicit visualization of the electron correlation, we uncover a coupled motion of the electrons that gives a new picture of bonding in molecules with extended conjugation, showing that even sys-

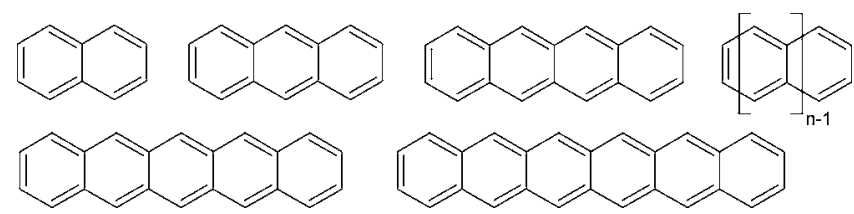

FIG. 1. The first few members and the unit cell of the acene series. 
tems such as the acenes can continue to provide fertile sources of surprising electronic structure.

\section{COMPUTATIONAL METHODOLOGY}

In the present context, we can regard the DMRG as an efficient way to exactly correlate, in the sense of full configuration interaction, the electrons in the active space. Details of the DMRG algorithm as implemented in our BLOCK code are given in Refs. 31-33. Active space full configuration interaction theory is sometimes referred to as CASCI. Recall that CASCI is the same as the more common complete active space self-consistent field (CASSCF) method ${ }^{24}$ but lacks the step of orbital optimization. Orbital optimization is possible within the DMRG but has not been used here. For the molecules in this work, the DMRG energies are converged to better than $0.1 \mathrm{kcal} / \mathrm{mol}$ and would be identical to the so-called CASCI energies if it were possible to compute these in the traditional manner.

DMRG calculations on the acenes were performed at the UB3LYP/6-31G $(d)$ (Refs. 34 and 35) optimized singlet and triplet geometries which were essentially the same as those used by Bendikov et al. ${ }^{12}$ (see supporting information for details). These structures have $D_{2 h}$ point-group symmetry. The rung $\mathrm{C}-\mathrm{C}$ bonds are somewhat longer than the ladder $\mathrm{C}-\mathrm{C}$ bonds and the ladder $\mathrm{C}-\mathrm{C}$ bonds display increasing bond alternation toward the ends of the chain. For example, in singlet decacene, the rung and ladder $\mathrm{C}-\mathrm{C}$ bonds were 1.464 and $1.405 \AA$, respectively, at the middle of the chain, while the difference in successive ladder $\mathrm{C}-\mathrm{C}$ bonds lengths was $0.058 \AA$ at the end of the chain as compared to $0.010 \AA$ at the middle.

The active space was chosen to be the complete $\pi$-valence space, consisting of all conjugated carbon $p_{z}$ orbitals, and all $\pi$ electrons were correlated. The $\sigma$ electrons were treated within a frozen-core approximation using the restricted Hartree-Fock orbitals. The calculations used either the minimal STO-3G basis $^{36}$ (up to dodecacene) or Dunning's double- $\zeta$ DZ basis ${ }^{37,38}$ (up to hexacene) as indicated. In the case of the DZ basis, two $p_{z}$ orbitals per carbon were used to make a "double" complete $\pi$-valence space. Thus whereas, e.g., the DMRG/STO-3G calculations for pentacene correspond to a $(22,22)$ CASCI, the DMRG/DZ calculations would correspond to a $(22,44)$ CASCI. $^{82}$

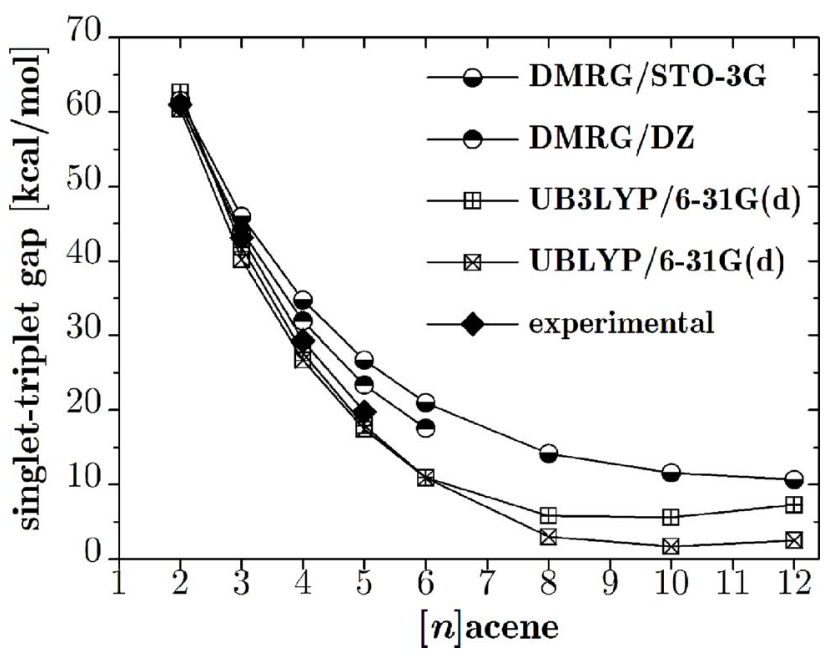

FIG. 2. Singlet-triplet energy gap as a function of the acene length.

\section{THE SINGLET-TRIPLET GAP}

In Fig. 2 we present the computed DMRG singlet-triplet energy gaps as a function of the acene length. The calculations on dodecacene correspond to a $(50,50)$ CASCI and are only made possible through the DMRG algorithm. Included for comparison are the UB3LYP/6-31G $(d)$ and UBLYP/6-31G $(d)$ singlet-triplet gaps (using open-shell wave functions where stable) as first reported by Bendikov et al., ${ }^{12}$ which we have recomputed and extended to the complete set of acenes studied here ${ }^{39}$ (Table I). While experimental triplet energies are somewhat difficult to compare directly with theoretical gas phase calculations, we have also included current experimental estimates where available. $^{40-43,83}$

Our DMRG calculations clearly confirm that the acenes maintain a singlet ground-state configuration and that there is a finite singlet-triplet gap for all chain lengths. Going from the minimal STO-3G to the DZ basis and the corresponding larger double-active space, the singlet-triplet gap decreases by a few $\mathrm{kcal} / \mathrm{mol}$. With the DZ basis the hexacene DMRG gap is $17.5 \mathrm{kcal} / \mathrm{mol}$. The remaining error in the DMRG calculations arises from the neglect of dynamical and $\sigma-\pi$ correlations, which would generally further decrease the gap

TABLE I. Singlet-triplet gap $\left(E_{\text {triplet }}-E_{\text {singlet }}\right)$ energies in $\mathrm{kcal} / \mathrm{mol}$ for the acene series.

\begin{tabular}{cccccc}
\hline \hline$[n]$-acene & DMRG/STO-3G & DMRG/DZ & UB3LYP/6-31G $(d)$ & UBLYP/6-31G $(d)$ & Expt. \\
\hline 2 & 61.5 & 61.0 & 62.6 & 60.4 & $61.0^{\mathrm{a}}$ \\
3 & 45.9 & 44.0 & 41.8 & 40.2 & $43.1^{\mathrm{b}}$ \\
4 & 34.7 & 31.9 & 27.7 & 26.7 & $29.3^{\mathrm{c}}$ \\
5 & 26.7 & 23.4 & 17.9 & 17.4 & $19.8^{\mathrm{d}}$ \\
6 & 21.0 & 17.5 & 10.9 & 10.9 & \\
8 & 14.2 & & 5.8 & 3.0 & \\
10 & 11.6 & & 5.6 & 1.7 & \\
12 & 10.7 & 7.3 & 2.5 & \\
\hline \hline
\end{tabular}

\footnotetext{
Reference 40 .

${ }^{\mathrm{b}}$ Reference 41.

${ }^{\mathrm{c}}$ Reference 42.

${ }^{\mathrm{d}}$ Reference 43.
} 
TABLE II. Effect of active space size and dynamical correlation on the singlet-triplet gap in smaller acenes. Complete $=$ complete $\pi$-valence space, double $=$ double $\pi$-valence space, partial $=$ incomplete active space: 2 -acene $(8,8), 3$ - and 4-acenes $(12,12)$. DZP $=$ Dunning DZ basis with polarization functions (Refs. 37 and 38) except for results of Kawashima et al. (Ref. 25). All energies in $\mathrm{kcal} / \mathrm{mol}$.

\begin{tabular}{lccc}
\hline \hline$[n]$-acene & 2 & 3 & 4 \\
\hline Complete/DZ & & & \\
CASSCF & 61.1 & & \\
CASPT2 & 60.5 & & \\
Complete/DZP & & & \\
CASSCF & 61.1 & & \\
CASPT2 & 59.7 & & $47.3^{\mathrm{a}}$ \\
Partial/DZP & & & $34.8^{\mathrm{a}}$ \\
CASSCF & 67.1 & $60.0^{\mathrm{a}}$ & \\
CASPT2/MRMP & 56.9 & $46.1^{\mathrm{a}}$ & 34.7 \\
Complete/STO-3G & & & 31.9 \\
DMRG & 61.5 & 45.9 & 29.3 \\
Double/DZ & & & \\
DMRG & 61.0 & 44.0 & \\
Expt. & 61.0 & 43.1 & \\
\hline \hline
\end{tabular}

${ }^{a}$ CASSCF/MRMP calculations of Kawashima et al. (Ref. 25); vertical singlet-triplet gap in a cc-pVDZ basis without polarization functions on $\mathrm{H}$.

size. However, we estimate the effect of dynamical correlation on the gap to be very small when using the complete (and the double complete) $\pi$-valence space, on the order of a few $\mathrm{kcal} / \mathrm{mol}$. In Table II, we present additional CASSCF and CASPT2 results ${ }^{44}$ (including the CASSCF and MRMP calculations of Kawashima et $a l_{.}^{25}$ ) for the smaller acenes to estimate the effects of dynamical correlation. CASPT2 (Ref. 45) and MRMP (Ref. 46) both incorporate dynamical correlation on top of the CASSCF reference through second-order perturbation theory. We observe in naphthalene that when using a complete $\pi$-valence space the CASSCF singlettriplet gap is very close (within $1-2 \mathrm{kcal} / \mathrm{mol}$ ) to the CASPT2 singlet-triplet gap. It is only when incomplete active spaces are used that the CASPT2/MRMP gap is significantly different from the CASSCF gap. In all cases, the DMRG complete and double $\pi$-valence space gaps are closer to the experimental result than the MRMP gap in an incomplete active space. This highlights the importance of the complete $\pi$-valence space for $\pi$-electron excitations.

Comparison of the UBLYP and UB3LYP gaps with the experimental data suggests that the DFT results are an underestimate. This is particularly true for UBLYP which substantially underestimates the gap. Surprisingly, the DFT gaps appear to increase between 10-acene and 12-acene.

Using our DMRG data we can extrapolate to the infinite polyacene limit. Empirically, we find that the singlet-triplet gap is well fitted by an exponential form $a+b e^{-c}$, giving a gap for the infinite chain of $8.69 \pm 0.95$ (STO-3G) and $3.33 \pm 0.39(\mathrm{DZ}) \mathrm{kcal} / \mathrm{mol}$, respectively, somewhat lower than the previous estimate of $12.2 \mathrm{kcal} / \mathrm{mol}$ obtained by Raghu et $a l .{ }^{30}$ using the semiempirical Pariser-Parr-Pople (PPP) Hamiltonian. ${ }^{47,48}$

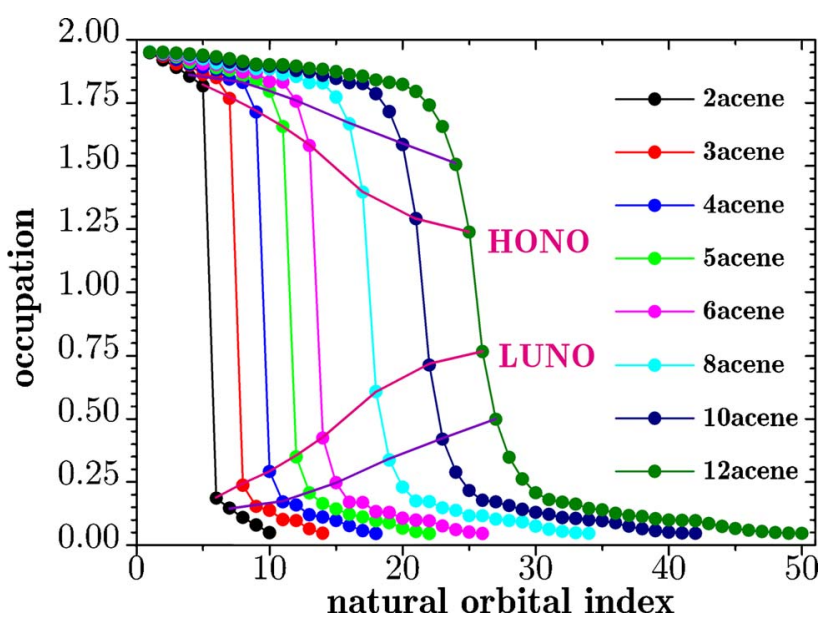

FIG. 3. Natural orbital occupation numbers for the acene series in the STO-3G basis. The lines are guides for the eye to show the evolution of the occupation numbers for the near singly occupied orbitals as a function of chain length.

\section{POLYRADICAL CHARACTER OF THE GROUND STATE}

Having established that the acene ground states are singlets, are they then singlet diradicals as argued by Bendikov et al. $?^{12} \mathrm{~A}$ simple way to establish whether there are unpaired electrons in a correlated wave function is to examine the occupation numbers of the (spinless) natural orbitals; in a closed-shell configuration, these are always 2 (doubly occupied) or 0 (unoccupied), while values close to 1 indicate single occupancy and unpaired electrons. ${ }^{49}$ In Fig. 3 we plot the occupancies of the natural orbitals for the acene series. We have designated the two orbitals with occupancies closest to 1 the "highest occupied natural orbital" (HONO) with occupancy greater than 1 and "lowest unoccupied natural orbital" (LUNO) with occupancy less than 1, respectively. These natural orbitals together with usual highest occupied molecular orbital (HOMO) and lowest unoccupied molecular orbital (LUMO) are shown in Fig. 4.

As can be seen, as we proceed to longer acenes the occupancies of the HONO and LUNO indeed approach 1, which is consistent with the prediction of Bendikov et al. of diradical character. The DZ basis, while yielding less radical character (e.g., the occupancies of the HONO in pentacene are 1.66 and 1.73 using the STO-3G and DZ bases, respectively), shows the same general behavior (the decreased radical character in the DZ basis is consistent with the general observation that radical character is reduced by dynamic correlation). However, what is surprising is that if we follow the trend for the next nearest single occupancy orbitals (the HONO-1 and the LUNO+1), the rate at which they approach single occupancy is comparable to that of the HONO and LUNO. This suggests that if we were to proceed to acenes longer than the 12-acene, we would eventually find not a diradical ground state but a polyradical ground state.

Several different measures of the number of "effectively unpaired" electrons in a molecule have previously been proposed. While such integrated measures must contain less information than the underlying distribution of natural orbital occupations examined above, we include them here for com- 

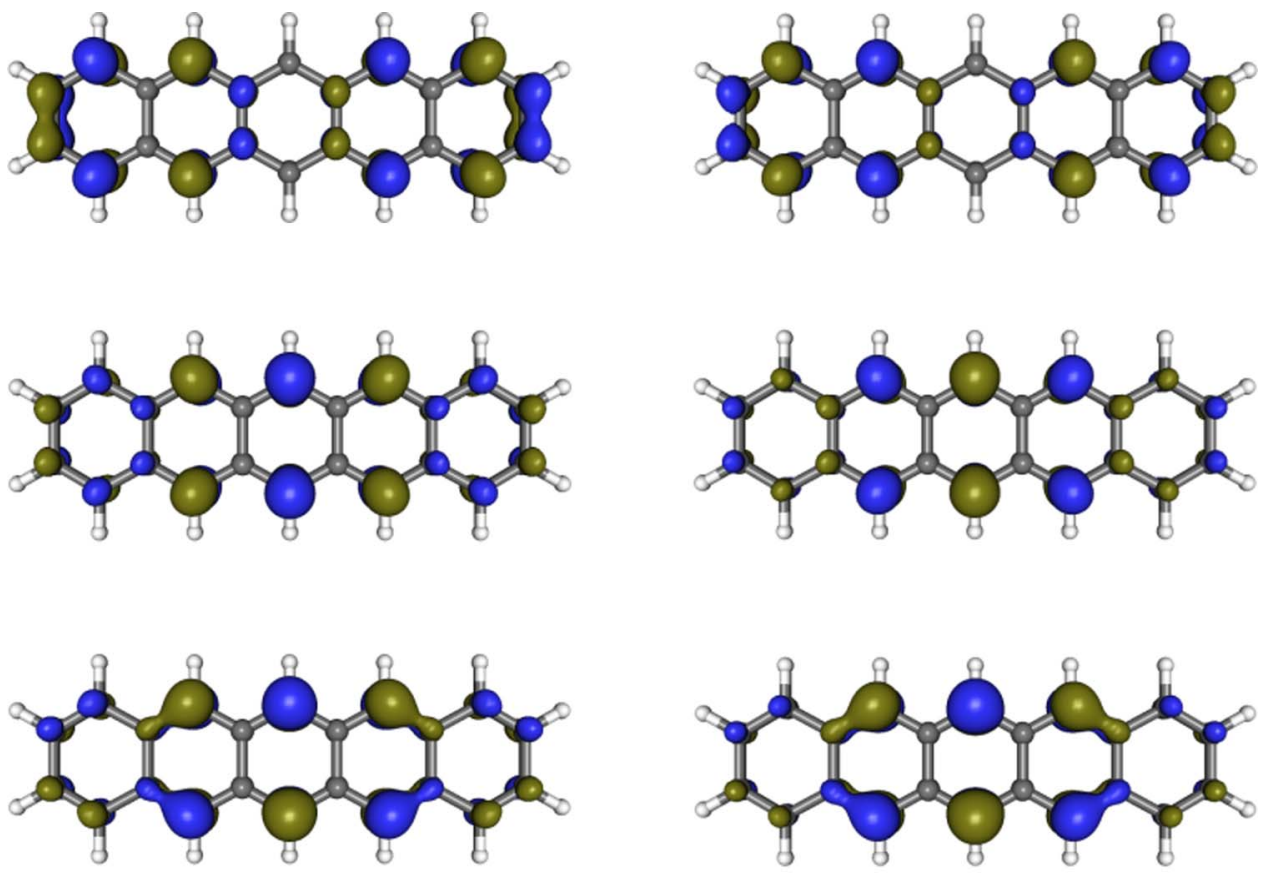

FIG. 4. Plots of the molecular orbitals (left: LUMO+1, LUMO, HOMO, $\mathrm{HOMO}-1$ ) and natural orbitals (right: LUNO+1, LUNO, HONO, HONO

$-1)$ for pentacene.
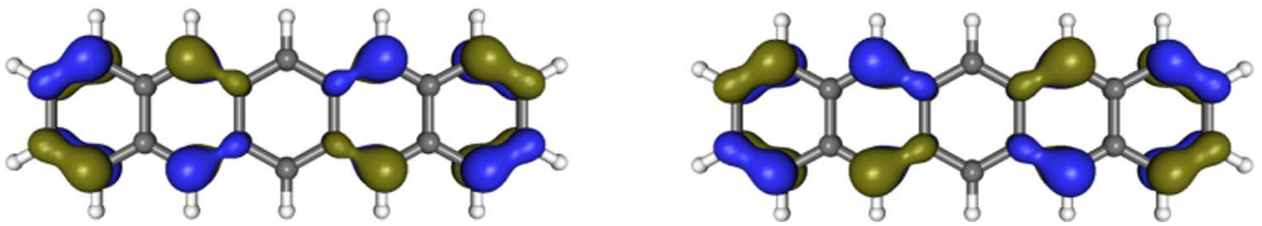

pleteness. We have investigated two measures, due to Takatsuka $^{50-54}$ and Head-Gordon, respectively, ${ }^{55-57}$ defined via

$$
\begin{aligned}
& N_{\text {unpaired }}^{\text {Takatsuka }}=\sum_{i} 2 n_{i}-n_{i}^{2}, \\
& N_{\text {unpaired }}^{\text {Head-Gordon }}=\sum_{i} \min \left(n_{i}, 2-n_{i}\right) .
\end{aligned}
$$

Here $n_{i}$ is the occupation number of the $i$ th natural orbital, which ranges from 0 to 2 . The contribution from each orbital is a maximum when $n_{i}=1$, whence each orbital contributes one electron to the effective number of unpaired electrons. These measures are plotted for the acenes in Fig. 5.

Some care must be taken when interpreting Fig. 5. Certainly, the values must not be taken literally; 12-acene does not contain 14 unpaired electrons. Both measures are extensive, meaning that they increase with the size of the molecule. (This also means that a large enough assembly of nearly closed-shell molecules would appear to have a substantial number of unpaired electrons using these measures. In such a case, however, the HONO and LUNO occupation numbers would not change and would stay near 2 and 0 as the number of molecules is increased, unlike what we see in the acenes.) However, extensive scaling should only be observed for system sizes larger than the typical size of an unpaired electron. Examination of the gradients of the plots in Fig. 5 shows an onset of extensive scaling around hexacene, which is consistent with the observation of Bendikov et al. that the first symmetry breaking of the density functional calculations occurs also at this chain length. This further suggests that we can roughly associate one unpaired spin with every five to six rings.

\section{VISUALIZING ELECTRON CORRELATIONS}

\section{A. Theoretical background}

Let us then regard the ground state of the longer acenes as being a singlet polyradical. How are we to understand its electronic structure? We can visualize the simultaneous behavior of the multiple electrons involved in the polyradical behavior through their correlation functions. Correlation functions have long been used to understand bond alternation and electron correlation in conjugated systems. ${ }^{29,58}$ We have evaluated three correlation functions, the particle-particle, spin-spin, and singlet diradical correlation functions which we now describe. In this section, we shall be concerned with the correlations of the electrons in real space. Thus, in the following, indices $i$ and $j$ always refer to the (orthogonalized) $p_{z}$ atomic orbitals on atoms $i$ and atom $j$, respectively. $\left\langle n_{i}^{\sigma}\right\rangle$ and $\left\langle S_{i}^{z}\right\rangle$ refer to the average $\sigma$ occupancy and $z$ component of the spin in these orbitals. 


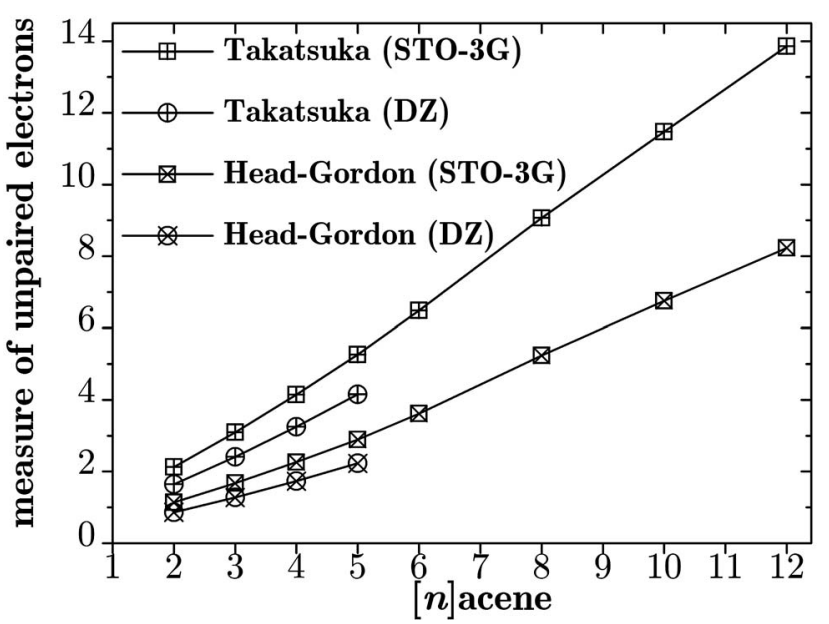

FIG. 5. Measures of the number of unpaired electrons in the acene series. See comment in text.

\section{Particle-particle:}

$$
C_{\text {particle }}(i, j)=4\left(\left\langle n_{i}^{\alpha} n_{j}^{\beta}\right\rangle-\left\langle n_{i}^{\alpha}\right\rangle\left\langle n_{j}^{\beta}\right\rangle\right) .
$$

This measures the correlation between the $\alpha$ population of orbital $i$ and $\beta$ population of orbital $j$. In a single determinant wave function (such as the Kohn-Sham wave function), there are no $\alpha \beta$ correlations and this quantity is identically zero.

Spin-spin:

$$
C_{\text {spin }}(i, j)=4\left(\left\langle S_{i}^{z} S_{j}^{z}\right\rangle-\left\langle S_{i}^{z}\right\rangle\left\langle S_{j}^{z}\right\rangle\right) .
$$

This measures the correlation between the spin in orbital $i$ and the spin in orbital $j$. Note that in wave functions that preserve the correct singlet-spin symmetry as used in this work, $\left\langle S_{i}^{z}\right\rangle=\left\langle S_{j}^{z}\right\rangle=0$. Because there are $\alpha \alpha$ and $\beta \beta$ correlations from the Pauli principle even in single determinant wave functions, this quantity does not fully vanish in noninteracting systems.

Singlet diradical:

$$
\begin{aligned}
& C_{\text {diradical }}(i, j)=2\left(\left\langle d_{i}^{\alpha} d_{j}^{\beta}\right\rangle-\left\langle d_{i}^{\alpha}\right\rangle\left\langle d_{j}^{\beta}\right\rangle+\left\langle d_{i}^{\beta} d_{j}^{\alpha}\right\rangle-\left\langle d_{i}^{\beta}\right\rangle\left\langle d_{j}^{\alpha}\right\rangle\right), \\
& d_{i}^{\alpha}=n_{i}^{\alpha}\left(1-n_{i}^{\beta}\right) .
\end{aligned}
$$

The single occupancy operator $d_{i}^{\alpha}$ measures the probability that an orbital $i$ is occupied with $\alpha$ spin without any simultaneous $\beta$ occupancy. This and the joint diradical probability density $\left\langle d_{i}^{\alpha} d_{j}^{\beta}\right\rangle$ were introduced by Dutoi et al. ${ }^{59}$ The function $C_{\text {diradical }}$ above is obtained by removing the independent probabilities of single occupation (e.g., $\left\langle d_{i}^{\alpha}\right\rangle\left\langle d_{j}^{\beta}\right\rangle$ ) from the probability density of Dutoi et al., to give the correlation between single occupancies of orbitals $i$ and orbital $j$ with opposite spins. Again, because of Pauli type correlations in single determinant wave functions, this quantity does not fully vanish in noninteracting systems.

To make the meanings of these correlation functions explicit, we can examine the following limiting cases for twoelectron wave functions $\Psi$. (Here $\phi_{1}$ and $\phi_{2}$ are disjoint orthogonal atomic orbitals.)

(1) Singlet diradical $\Psi=\frac{1}{2}\left(\phi_{1} \phi_{2}+\phi_{2} \phi_{1}\right)(\alpha \beta-\beta \alpha)$. In this case, the above correlation functions assume their extremum values of 1 or -1 . Thus we would find
$C_{\text {particle }}(1,1)=C_{\text {particle }}(2,2)=-1, \quad C_{\text {spin }}(1,1)=C_{\text {spin }}(2,2)$

$=1, \quad C_{\text {diradical }}(1,1)=C_{\text {diradical }}(2,2)=-1, \quad$ and $C_{\text {particle }}(1,2)=1, C_{\text {spin }}(1,2)=-1$, and $C_{\text {diradical }}(1,2)=1$.

(2) Closed-shell singlet $\Psi=\frac{1}{2 \sqrt{2}}\left(\phi_{1}+\phi_{2}\right)\left(\phi_{1}+\phi_{2}\right)(\alpha \beta-\beta \alpha)$. In this case all correlation functions are identically 0 , reflecting the absence of $\alpha \beta$ correlation.

(3) Triplet $m_{s}=0$ diradical $\Psi=\frac{1}{2}\left(\phi_{1} \phi_{2}-\phi_{2} \phi_{1}\right)(\alpha \beta+\beta \alpha)$. Here all correlation function values are identical to those for the singlet diradical wave function. (One observes that the $m_{s}=0$ triplet wave function differs from that of the singlet diradical only in the phase relationship between orbital products such as $\phi_{1}^{\alpha} \phi_{2}^{\beta}$ and $\phi_{1}^{\beta} \phi_{2}^{\alpha}$, thus to distinguish the two one should examine averages such as $\left\langle S_{i}^{+} S_{j}^{-}\right\rangle$. Note that $m_{s}=1$ triplet states were used in this study.)

\section{B. Correlation functions}

Figures 6 and 7 show plots of the different correlation functions evaluated for the singlet ground states of napthalene, pentacene, and dodecacene. Since the correlation functions are functions of two positions, we have plotted them as a function of the second position with the first (reference) position fixed (indicated by the boxed value in the figures).

In Fig. 6 all plots have the reference position fixed at the center of the lower acene strand. Examining $C_{\text {particle }}$ we see that it is large and negative at the reference position. Thus given an $\alpha$ electron in this orbital, there is a significantly decreased chance of finding a simultaneous $\beta$ occupation of the orbital, or more simply, double occupancy of the atomic orbital is disfavored. Moving one atom away, $C_{\text {particle }}$ is large and positive reflecting an increased chance of finding the orbital to be occupied with opposite spin to that at the reference position. This antiferromagnetic correlation continues further away from the reference position in a pattern of positive and negative values of $C_{\text {particle }}$, though the rapidly decreasing amplitudes indicate that the correlations are short ranged.

Examining the spin-spin $C_{\text {spin }}$ and singlet diradical $C_{\text {diradical }}$ correlation functions yields a similarly consistent picture. $C_{\text {spin }}$ is large and positive at the reference position while $C_{\text {diradical }}$ is large and negative, which both indicate that the orbital has a strong tendency toward single occupation. The neighboring atoms further show strong single occupancy, antiferromagnetic correlation with large negative (positive) values of $C_{\text {spin }}\left(C_{\text {diradical }}\right)$, and this correlation decreases rapidly further away from the reference position. While $C_{\text {spin }}$ and $C_{\text {diradical }}$ do not identically vanish for a single determinant uncorrelated wave function, their corresponding plots for pentacene in Fig. 6 show that aside from a small reduced propensity for double occupancy at the reference position which results from electron delocalization, there are no significant antiferromagnetic correlations along the chains. (Recall that $C_{\text {particle }}$ is identically zero in the single determinantal wave function.)

Comparing the correlation functions of napthalene and dodecacene shown in Fig. 6, for which the reference atom is in both cases at an "inner" position on the strand, we see that there is a (slight) increase in the antiferromagnetic correla- 
(a)
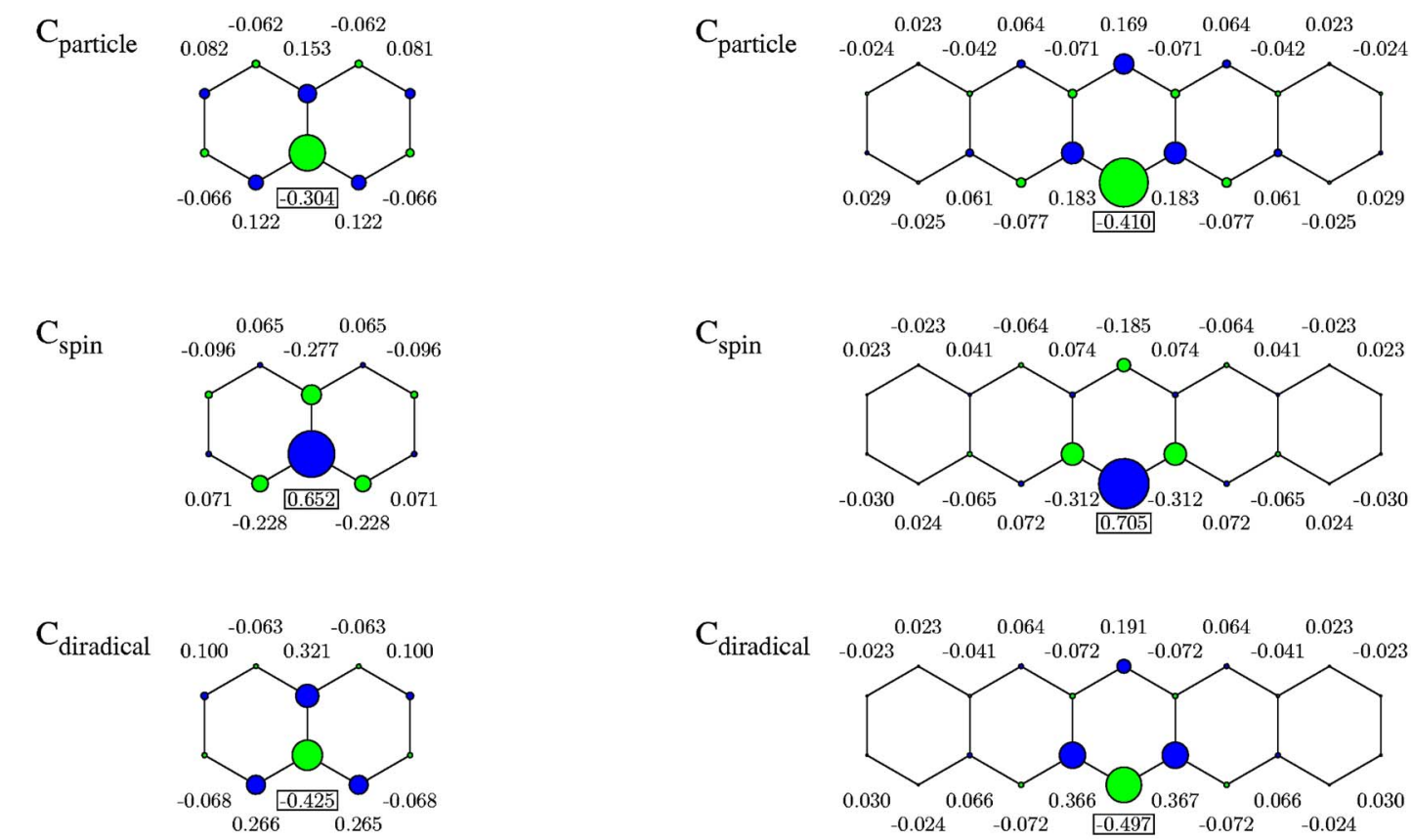

$\mathrm{C}_{\text {spin }}$
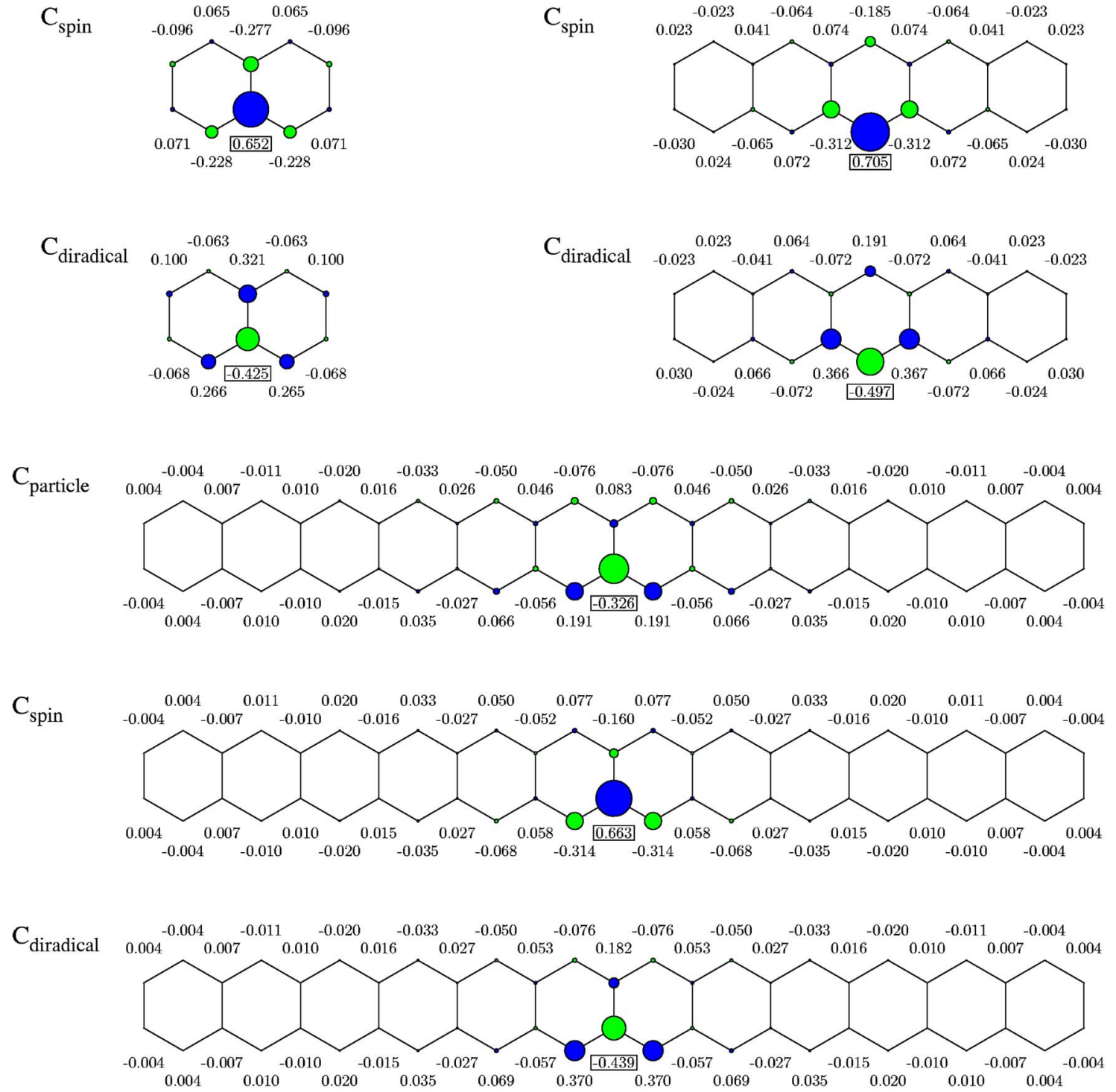

(b)
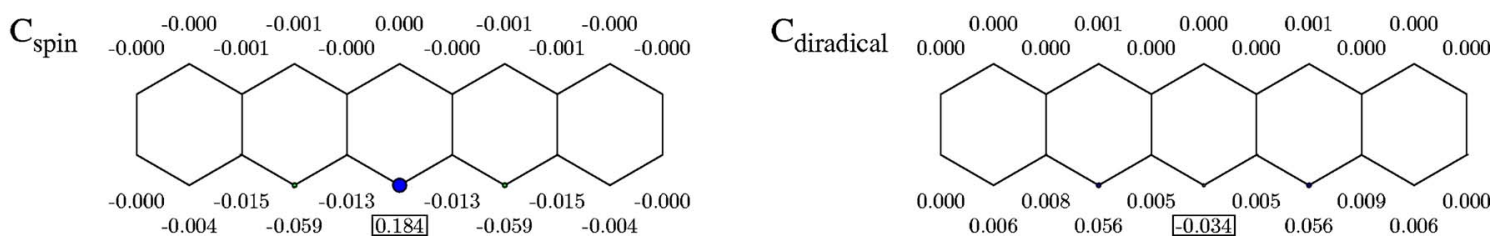

FIG. 6. (a) Particle-particle, spin-spin, and singlet diradical correlation functions evaluated for napthalene, pentacene, and dodecacene in the STO-3G basis, fixing the reference point at the center of the lower acene strand (indicated by the boxed value). The value of the correlation function is indicated by the numbers; the size and color of the circles give the magnitude and sign of the correlation function, respectively. (b) Correlation functions in a noninteracting model of pentacene. 

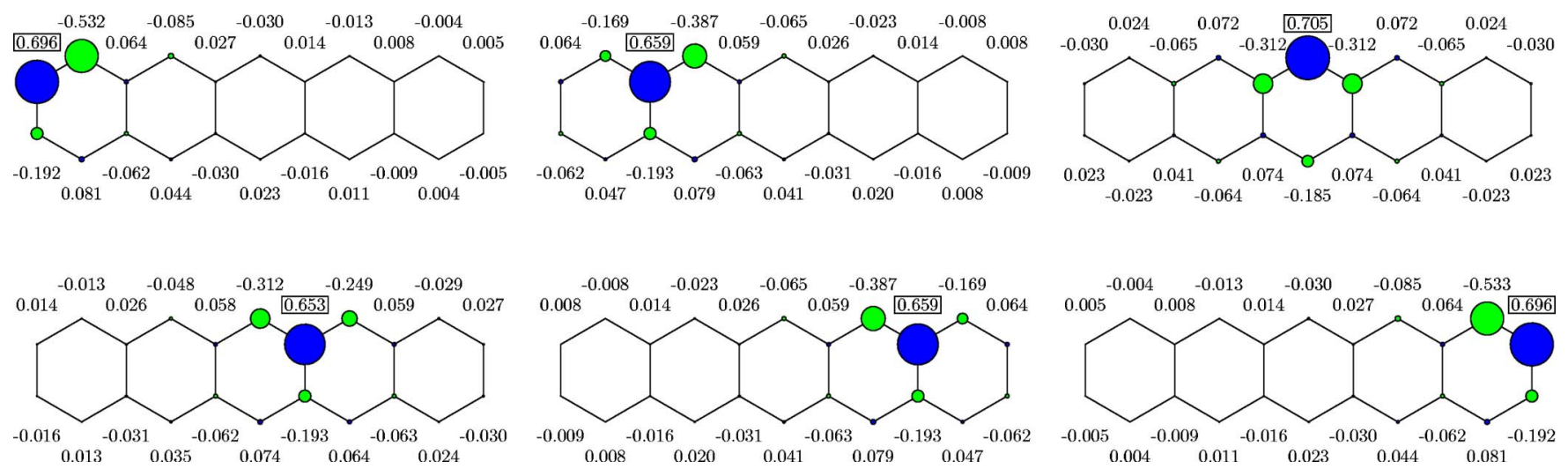

FIG. 7. Spin-spin correlation functions for pentacene as we move the reference point of the correlation function (indicated by the boxed value) around the ring.

tions as the length of the acene increases. In napthalene the correlation between the reference atom and the atom on the neighboring strand is stronger than the correlation to its neighbors on the same strand, a situation which is reversed in the longer acenes. This is consistent with the increasing difference between the ladder and rung $\mathrm{C}-\mathrm{C}$ bond-lengths, which leads to the view of the longer acenes as a pair of coupled polyacetylene strands. ${ }^{11,12}$

Figure 7 shows the spin-spin correlation plots where we move the reference position around the ring. As expected the antiferromagnetic correlations persist as the reference position is moved. Bond alternation is stronger near the edges of the pentacene ring and this leads to asymmetrical correlations between the reference position and its neighbors; stronger correlations are observed across the shorter bonds.

\section{THE NATURE OF BONDING IN THE ACENE POLYRADICAL STATE}

The correlation functions evaluated above present a dynamic picture of the electronic motion in the acenes. Tracking a single electron as it makes its way around the ring, a second electron is pulled along, antiferromagnetically coupled to the first and distributed over the nearest neighbor atoms.

Short-range antiferromagnetic correlations naturally bring to mind resonating valence bonds. ${ }^{60-65}$ Recall that we can expand any wave function in terms of resonance structures, which may be classified as covalent, singly ionic, doubly ionic, and so on (see Fig. 8). (In this language, the resonance structures are viewed only as a many-body basis for expanding the wave function; the molecular geometry is fixed across the different structures.) From our correlation functions we see that the acene ground state is dominated by covalent resonance structures (no double occupancy of the $p_{z}$

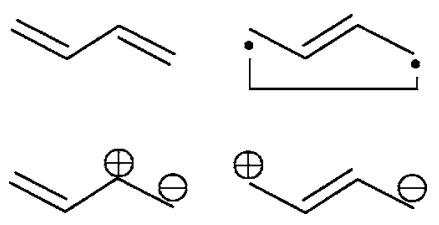

FIG. 8. Covalent (top) and ionic (bottom) resonance structures for a conjugated system. Note that electron delocalization requires a combination of both covalent and ionic resonance structures with roughly equal weights. orbitals) with short-range spin couplings (i.e., short-ranged antiferromagnetic correlations).

We should note that the use of the word resonance here is different from the colloquial usage where resonance structures are a simple metaphor for delocalization. In terms of the resonance structures as a physical basis, electron delocalization requires superposition of covalent and ionic structures with roughly equal weights. In the limit where the wave function is comprised only of covalent structures, we instead have an extreme localization: The electrons are fixed and unpaired on each of the atomic sites with only a spin degree of freedom, which fluctuates between different kinds of spin couplings. Wave functions which are predominantly covalent in nature can therefore be viewed as polyradicals as every electron is unpaired in a localized, isoenergetic atomic orbital. The covalent nature of the acene ground state revealed by the correlation functions argues for this polyradical interpretation, which is consistent with the picture given earlier by the natural orbital occupations.

Valence bond language in conjugated $\pi$ systems has long been appreciated in the context of their low-lying states, which are conventionally classified as covalent or ionic depending on the main resonance structures. Typically, covalent states appear more naturally described in the valence bond language. For example, in the polyenes, which may be thought of as making the two legs of the acene ladder, the lowest excitation is a covalent $2 A_{g}$ state which appears to have large double excitation character from a molecular orbital viewpoint. However, this low-lying double excitation is easily understood in the valence bond language as arising from the singlet recoupling of two singlet $\rightarrow$ triplet excitations on adjacent double bonds. ${ }^{66-71}$ Valence bond descriptions and analyses have also been examined in the context of radical electronic structure. ${ }^{18,72}$ In traditional CAS calculations, to extract a valence bond picture one usually reparametrizes the wave function through a valence bond expansion [sometimes known as CASVB (Refs. 73-77)]. Such studies also find that the benzene ground state should be viewed as a covalent state with antiferromagnetic spin couplings, in accordance with what we have found for the acenes. The exponential size of the valence bond basis limits the CASVB analysis to small molecules, but as we have demonstrated, correlation functions provide an alternative mechanism to infer the resonance nature of a state. 
In a simple view of bonding, such as that afforded by the Hubbard $^{78,79}$ or Pariser-Parr-Pople models, there are two scales of energy, the resonance or hopping energy $t$ associated with delocalization and the Coulomb repulsion energy $U$ associated with double occupancy of an atomic orbital. When $U / t \gg 1$, we may be said to be in the strongly interacting regime. Under such circumstances, the molecular orbital picture begins to break down and instead the appropriate qualitative wave function is the superposition of covalent resonance structures as described above. A standard choice of parameters for conjugated polymers in the Ohno parametrization of the PPP Hamiltonian is $U=11.26 \mathrm{eV}$ and $t$ $=2.4 \mathrm{eV},{ }^{29,80,81}$ placing systems such as the acenes in the moderately strongly interacting spectrum of Hamiltonians and therein lies an understanding of the polyradical character and covalent ground state that we have observed.

\section{CONCLUSIONS}

In summary, motivated by predictions of unusual ground states in the longer acene molecules, we investigated acene electronic structure with high-level wave function theory. Using a new $a b$ initio density matrix renormalization group algorithm we could carry out complete active space calculations on the acenes from napthalene to dodecacene that correlated the full $\pi$-valence space. We find that the ground state remains a singlet as the chain length increases, with a finite singlet-triplet gap in the infinite chain limit. Detailed examination of the wave functions, natural orbitals, and effective number of unpaired electrons further reveals that the longer acenes exhibit singlet polyradical character in their ground state. Through a series of correlation functions we observe that electrons are antiferromagnetically coupled in pairs on neighboring atoms as they move around the acene chains. These results are consistent with a view of the longer acenes as moderately strongly interacting electronic systems, for which the appropriate reference description is a polyradical wave function arising from a resonance of predominantly covalent valence bond structures. We note that such a viewpoint is essential to understand the excitations of these systems. Finally, our study illustrates that even simple systems such as the acenes can provide unusual surprises in their electronic structure.

\section{ACKNOWLEDGMENTS}

One of the authors (J.H.) is funded by a Kekulé Fellowship of the Fond der Chemischen Industrie (Fund of the German Chemical Industry). Another author (M.A.) was supported by the Cornell Center for Materials Research (CCMR) through their REU program. Another auther (G.K.L.C.) acknowledges support from Cornell University, CCMR, the David and Lucile Packard Foundation in Science and Engineering, and the National Science Foundation CAREER program CHE-0645380.

\footnotetext{
${ }^{1}$ E. Clar, Polycyclic Hydrocarbons (Academic, London, 1964).

${ }^{2}$ R. G. Havey, Polycyclic Aromatic Hydrocarbons (Wiley-VCH, New York, 1997).

${ }^{3}$ Y. Geerts, G. Klärner, and K. Müllen, in Electronic Materials: The Oligomer Approach, edited by K. Müllen and G. Wagner (Wiley-VCH,
}

Weinheim, 1998), p. 48.

${ }^{4}$ C. D. Dimitrakopoulos and P. R. L. Malenfant, Adv. Mater. (Weinheim, Ger.) 14, 99 (2002).

${ }^{5}$ C. Reese, M. Roberts, M. Ling, and Z. Bao, Mater. Today 7, 20 (2004). ${ }^{6}$ M. Bendikov, F. Wudl, and D. F. Perepichka, Chem. Rev. (Washington, D.C.) 104, 4891 (2004).

${ }^{7}$ H. Angliker, E. Rommel, and J. Wirz, Chem. Phys. Lett. 87, 208 (1982).

${ }^{8}$ M. Kertesz and R. Hoffmann, Solid State Commun. 47, 97 (1983).

${ }^{9}$ S. Kivelson and O. L. Chapman, Phys. Rev. B 28, 7236 (1983).

${ }^{10}$ K. B. Wiberg, J. Org. Chem. 62, 5720 (1997).

${ }^{11}$ K. N. Houk, P. S. Lee, and M. Nendel, J. Org. Chem. 66, 5517 (2001).

${ }^{12}$ M. Bendikov, H. M. Duong, K. Starkey, K. N. Houk, E. A. Carter, and F. Wudl, J. Am. Chem. Soc. 126, 7416 (2004); ibid. 126, 10493 (2004).

${ }^{13}$ R. Mondal, B. K. Shah, and D. C. Neckers, J. Am. Chem. Soc. 128, 9612 (2006).

${ }^{14}$ A. R. Reddy and M. Bendikov, Chem. Commun. 1179 (2006).

${ }^{15}$ T. Bally and W. T. Borden, Rev. Comput. Chem. 13, 1 (1999).

${ }^{16}$ J. F. Stanton and J. Gauss, Adv. Chem. Phys. 125, 101 (2003).

${ }^{17}$ L. V. Slipchenko and A. I. Krylov, J. Chem. Phys. 117, 4694 (2002).

${ }^{18}$ L. Salem and C. Rowland, Angew. Chem., Int. Ed. 11, 92 (1972).

${ }^{19}$ W. T. Borden and E. R. Davidson, J. Am. Chem. Soc. 99, 4587 (1977).

${ }^{20}$ W. T. Borden, Diradicals (Wiley, New York, 1982).

${ }^{21}$ A. Rajca, Chem. Rev. (Washington, D.C.) 94, 871 (1994).

${ }^{22}$ Y. Jung and M. Head-Gordon, ChemPhysChem 4, 522 (2003).

${ }^{23}$ A. I. Krylov, J. Phys. Chem. A 109, 10638 (2005).

${ }^{24}$ B. O. Roos, Adv. Chem. Phys. 69, 399 (1987).

${ }^{25}$ Y. Kawashima, T. Hashimoto, H. Nakano, and K. Hirao, Theor. Chem. Acc. 102, 49 (1999).

${ }^{26}$ S. R. White, Phys. Rev. Lett. 69, 2863 (1992).

${ }^{27}$ S. R. White, Phys. Rev. B 48, 10345 (1993).

${ }^{28}$ S. R. White and R. L. Martin, J. Chem. Phys. 110, 4127 (1999).

${ }^{29}$ C. Raghu, Y. Anusooya Pati, and S. Ramasesha, Phys. Rev. B 65, 155204 (2002).

${ }^{30}$ C. Raghu, Y. Anusooya Pati, and S. Ramasesha, Phys. Rev. B 66, 035116 (2002).

${ }^{31}$ J. Hachmann, W. Cardoen, and G. K.-L. Chan, J. Chem. Phys. 125, 144101 (2006).

${ }^{32}$ G. K.-L. Chan and M. Head-Gordon, J. Chem. Phys. 116, 4462 (2002).

${ }^{33}$ G. K.-L. Chan, J. Chem. Phys. 120, 3172 (2004).

${ }^{34}$ C. Lee, W. Yang, and R. G. Parr, Phys. Rev. B 37, 785 (1988).

${ }^{35}$ A. D. Becke, J. Chem. Phys. 98, 5648 (1993).

${ }^{36}$ W. J. Hehre, R. F. Stewart, and J. A. Pople, J. Chem. Phys. 51, 2657 (1969).

${ }^{37}$ T. H. Dunning, Jr., J. Chem. Phys. 53, 2823 (1970).

${ }^{38}$ T. H. Dunning, Jr. and P. J. Hay, in Methods of Electronic Structure Theory, edited by H. F. Schaefer III (Plenum, New York, 1977), Vol. 2.

${ }^{39}$ M. J. Frisch, G. W. Trucks, and H. B. Schlegel, GAUSSIAN 03, Revision C.02, Gaussian, Inc., Wallingford, CT, 2004.

${ }^{40}$ J. B. Birks, Photophysics of Aromatic Molecules (Wiley, London, 1970).

${ }^{41}$ J. Schiedt and R. Weinkauf, Chem. Phys. Lett. 266, 201 (1997).

${ }^{42}$ N. Sabbatini, M. T. Indelli, M. T. Gandolfi, and V. Balzani, J. Phys. Chem. 86, 3585 (1982).

${ }^{43}$ J. Burgos, M. Pope, Ch. E. Swenberg, and R. R. Alfano, Phys. Status Solidi B 83, 249 (1977).

${ }^{44}$ MOLPRO, a package of ab initio programs designed by H.-J. Werner and P. J. Knowles, version 2002.6, R. D. Amos, A. Bernhardsson, A. Berning, et al.

${ }^{45}$ K. Andersson, P.-A. Malmqvist, and B. O. Roos, J. Chem. Phys. 96, 1218 (1992)

${ }^{46}$ K. Hirao, Chem. Phys. Lett. 190, 374 (1992).

${ }^{47}$ R. Pariser and R. G. Parr, J. Chem. Phys. 21, 466 (1953); ibid. 21, 767 (1953).

${ }^{48}$ J. A. Pople, Trans. Faraday Soc. 49, 1375 (1953).

${ }^{49}$ D. Döhnert and J. Koutecký, J. Am. Chem. Soc. 102, 1789 (1980).

${ }^{50}$ K. Takatsuka, T. Fueno, and K. Yamaguchi, Theor. Chim. Acta 48, 175 (1978).

${ }^{51}$ K. Takatsuka and T. Fueno, J. Chem. Phys. 69, 661 (1978).

${ }^{52}$ R. C. Bochicchio, J. Mol. Struct.: THEOCHEM 429, 229 (1998).

${ }^{53}$ V. N. Staroverov and E. R. Davidson, J. Am. Chem. Soc. 122, 186 (2000).

${ }^{54}$ V. N. Staroverov and E. R. Davidson, Chem. Phys. Lett. 330, 161 (2000).

${ }^{55}$ M. Head-Gordon, Chem. Phys. Lett. 372, 508 (2003).

${ }^{56}$ R. C. Bochicchio, A. Torre, and L. Lain, Chem. Phys. Lett. 380, 486 (2003). 
${ }^{57}$ M. Head-Gordon, Chem. Phys. Lett. 380, 488 (2003).

${ }^{58}$ G. Fano, F. Ortolani, and L. Ziosi, J. Chem. Phys. 108, 9246 (1998).

${ }^{59}$ A. D. Dutoi, Y. Jung, and M. Head-Gordon, J. Phys. Chem. A 108, 10270 (2004).

${ }^{60}$ D. Cooper, Valence Bond Theory (Elsevier, Amsterdam, 2002).

${ }^{61}$ W. A. Goddard III and L. B. Harding, Annu. Rev. Phys. Chem. 29, 363 (1978).

${ }^{62}$ J. Gerratt, D. L. Cooper, P. B. Karadakov, and M. Raimondi, Chem. Soc. Rev. 26, 87 (1997)

${ }^{63}$ S. Shaik and P. C. Hiberty, Rev. Comput. Chem. 20, 1 (2004).

${ }^{64}$ M. A. Garcia-Bach, A. Peñaranda, and D. J. Klein, Phys. Rev. B 45, 10891 (1992).

${ }^{65}$ Y. Gao, C.-G. Liu, and Y.-S. Jiang, J. Phys. Chem. A 106, 2592 (2002).

${ }^{66}$ K. Schulten and M. Karplus, Chem. Phys. Lett. 14, 305 (1972).

${ }^{67}$ T. H. Dunning, R. P. Hosteny, and I. Shavitt, J. Am. Chem. Soc. 95, 5067 (1973).

${ }^{68}$ I. Ohmine, M. Karplus, and K. Schulten, J. Chem. Phys. 68, 2298 (1978).

${ }^{69}$ B. S. Hudson, B. E. Kohler, and K. Schulten, in Excited States, edited by E. C. Lim (Academic, New York, 1982), Vol. 6, p. 1.

${ }^{70}$ M. Said, D. Maynau, and J. P. Malrieu, J. Am. Chem. Soc. 106, 580 (1984).

${ }^{71}$ W. Wu, D. Danovich, A. Shurki, and S. Shaik, J. Phys. Chem. A 104, 874 (2000).

${ }^{72}$ T. Wang and A. I. Krylov, J. Chem. Phys. 123, 104304 (2005).

${ }^{73}$ K. Hirao, H. Nakano, K. Nakayama, and M. Dupuis, J. Chem. Phys. 105, 9227 (1996).
${ }^{74}$ T. Thorsteinsson, D. L. Cooper, J. Gerratt, P. B. Karadakov, and M. Raimondi, Theor. Chim. Acta 93, 343 (1996).

${ }^{75}$ T. Thorsteinsson, D. L. Cooper, J. Gerratt, and M. Raimondi, in Quantum Systems in Chemistry and Physics: Trends in Methods and Applications: A New Approach to Valence Bond Calculations: CASVB, edited by R. McWeeny, J. Maruani, Y. G. Smeyers, and S. Wilson (Kluwer, Dordrecht, 1997).

${ }^{76}$ D. L. Cooper, T. Thorsteinsson, and J. Gerratt, Adv. Quantum Chem. 32, 51 (1998).

${ }^{77}$ T. Thorsteinsson and D. L. Cooper, in Quantum Systems in Chemistry and Physics: Basic Problems and Models Systems: An Overview of the CASVB Approach to Modern Valence Bond Calculations, edited by A. Hernández-Laguna, J. Maruani, R. McWeeny, and S. Wilson (Kluwer, Dordrecht, 2000), Vol. 1, p. 303.

${ }^{78}$ J. Hubbard, Proc. R. Soc. London, Ser. A 276, 238 (1963).

${ }^{79}$ E. H. Lieb and F. Y. Wu, Phys. Rev. Lett. 20, 1445 (1968).

${ }^{80}$ K. Ohno, Theor. Chim. Acta 2, 219 (1964).

${ }^{81}$ G. Klopman, J. Am. Chem. Soc. 86, 4550 (1964).

${ }^{82}$ See EPAPS Document No. E-JCPSA6-127-309732 for further details of the calculations, energies, optimized geometries for all calculated oligoacenes at UB3LYP/6-31G(d) level, the data for Fig. 5, and complete Ref. 39. This document can be reached via a direct link in the online article's HTML reference section or via the EPAPS homepage (http:// www.aip.org/pubservs/epaps.html).

${ }^{83}$ The hexacene "experimental" number reported in Ref. 11 is, in fact, the theoretical extrapolation given in Ref. 7 and is too low. 